\title{
Pôles de compétitivité et spécificité de la ressource technologique : une illustration grenobloise
}

\author{
Competitiveness poles and specification \\ of technologic resource : the case of Grenoble
}

\author{
Bernard Pecqueur
}

Univ. de Grenoble II, IGA, B.P. 53, 38041 Grenoble Cedex 09, France

\section{Résumé}

Ce papier propose une lecture théorique des pôles de compétitivité. Après en avoir rappelé les principales caractéristiques, l'auteur développe l'hypothèse d'une ville qui produit, devenue acteur majeur de la mondialisation et participant à l'épuisement supposé du fordisme. L'auteur propose de voir dans les rapports production/territoire l'émergence d'un nouveau capitalisme. Il défend l'idée que les fondements de productions de richesse évoluent et deviennent relationnels. Il ne faut pas dès lors attribuer au pôle un objectif de productivité mais plutôt de spécification.

(c) 2008 Lavoisier, Paris. Tous droits réservés.

\section{Summary}

This article proposes a theoretical reading of the competitiveness poles. After bringing back to mind their main features, the author makes the hypothesis of a producing city that has become a major actor of globalization and that participates in the assumed depletion of the fordist model. The author suggests to see the emergence of a new capitalism in the relation between production and territory. He defends the idea that the basis of wealth production evolve and become relational. Hence one should not assign to the pole a goal of productivity but rather of specification.

(c) 2008 Lavoisier, Paris. Tous droits réservés.

\footnotetext{
*Adresse email : pecqueur@ujf-grenoble.fr

doi:10.3166/ges.10.311-326 @ 2008 Lavoisier, Paris. Tous droits réservés.
} 
Mots clefs : Pôles de compétitivité, globalisation, spécification, nouveau capitalisme

Keywords: competitiveness poles, globalization, specification, new capitalism

\section{Introduction}

Depuis les années 1970, nous vivons un phénomène de globalisation renforcé qui s'exprime à travers l'intensification des échanges de différents types : informations, capitaux, biens et services, mais aussi par le déplacement de personnes. Les flux sont d'emblée mondiaux. Les théoriciens de la mondialisation comme le japonais Ohmae (1995), concèdent que les formes des échanges mondiaux passent par des processus de régionalisation à travers la constitution de vastes zones de libre-échange, sur fond de «triadisation ». L'organisation de l'économie mondiale se ferait autour de trois grands pôles de développement majeur : l'Amérique du Nord, l'Union Européenne et le Japon (la triade). Pourtant, dans la dynamique actuelle des sociétés et des économies où Mac Luhan (1970) voyait un "village planétaire », la recherche en sciences sociales (notamment en Italie avec les Districts Industriels) a découvert que les relations de proximité entre les acteurs locaux peuvent jouer un rôle déterminant dans la compétitivité des activités économiques. L'aggravation, dans les années 1970 et 1980, des déficits publics a incité les États à une décentralisation et à une régionalisation de la gestion des biens collectifs locaux, tels que les infrastructures de transports, l'éducation, la formation, voire les aides au développement et à l'insertion sociale des chômeurs.

On observe alors que la densité des relations entre les acteurs locaux (entreprises, municipalités, universités, centres de recherche,) jouent un rôle déterminant dans la compétitivité de certaines activités industrielles et de services. Les districts industriels italiens semblent avoir leur équivalent dans le Bade-Wurtenberg tout comme dans certaines préfectures japonaises, dans la Silicon Valley ou dans les Systèmes Productifs Localisés (SPL) français, mis en lumière par des chercheurs grenoblois et repris emblématiquement par la DATAR. Les politiques d'aménagement du territoire, à la charge du pouvoir central jusque dans les années quatre-vingts, ont également été déléguées aux collectivités territoriales. Le « développement local », se substitue désormais au développement «par le haut ». «Il n'y a pas de territoires en crise, il y a seulement des territoires sans projet », déclarait ainsi en 1997, Charles Pasqua, alors ministre français de l'Aménagement du Territoire. Cette approche est devenue incontournable aussi bien en économie qu'en politique. La prise en compte des facteurs locaux dans les dynamiques économiques apparaît aujourd'hui comme une évidence et une impérieuse nécessité. Il s'agit, somme toute, d'une préoccupation relativement récente, qui ouvre la voie vers la diversification des politiques économiques, sociales et culturelles, et incite à proposer de nouvelles modalités de coordination entre les acteurs. Le dialogue entre les entreprises et les pouvoirs publics locaux devient, s'il est réel, un avantage pour tous : pour l'entreprise qui trouve des externalités dans son engagement local et pour les acteurs habitants des lieux qui y trouvent une activité économique qui permet la vie sociale.

Le pôle de compétitivité s'inscrit donc dans les figures contemporaines de la construction de la globalisation. En ce sens il est différent du pôle de croissance (perrousien) et du 
pôle technologique (du type Sophia Antipolis). En effet, le pôle «perrousien » valorise la qualité de l'entreprise (amont ou aval de filière, type d'industrie, etc.) plutôt que la qualité du lieu (son histoire, ses spécificités) alors que le pôle technologique n'est qu'un pôle perrousien qui aurait muté vers de nouveaux savoir faire sans pour autant changer l'esprit du pôle proprement industriel. Le pôle de compétitivité, selon l'esprit de ses concepteurs, intègre une notion de « spécificité » qui le distingue des autres formes et l'arrime à des territoires sans l'éloigner des enjeux globaux.

L'objectif de cette contribution vise d'abord à situer ce type de pôle dans le contexte post fordiste sous un premier angle, celui de la ville. Le pôle de compétitivité reflète dans son fonctionnement le nouvel avatar urbain : la ville qui produit. Dans une seconde partie seront analysées les caractéristiques de fond qui définissent le projet de ce type de pôle sans préjuger des résultats effectifs. Ces caractéristiques sont doubles : la spécificité et la proximité géographique, et contribuent à la compréhension de la nature même de la globalisation dans la période contemporaine. Ces caractéristiques permettent d'établir une première esquisse d'un modèle urbain des pôles de compétitivité.

\section{Mutations des fonctions de la ville et spécificité des pôles de compétitivité}

Avant de développer notre hypothèse de la ville qui produit (Pecqueur, 2006), précisons la définition du pôle de compétitivité qui diffère des types de pôles qui lui ont précédé.

\subsection{Le pôle de compétitivité : un «cluster» pas comme les autres}

Les pôles de compétitivité (2005), du point de vue de la politique publique, font suite aux SPL (1997). Cette initiative confirme le croisement intime entre organisation industrielle, territoire et compétitivité économique, à partir d'un constat portant sur la compétitivité économique et industrielle de la France en Europe et dans le monde (Blanc, 2004).

Cette idée défendue par les économistes et des géographes étudiant les dynamiques territoriales, trouve donc aujourd'hui un écho. La nouvelle étape engagée en 2005 insère les notions de réseau, de cluster, de sous-systèmes industriels spécifiques au cœur même de la politique industrielle française et partiellement dans la politique européenne (encadré $n^{\circ} 1$, Grenoble : le pôle Minalogic).

\section{Grenoble : le pôle MINALOGIC :}

Le pôle proprement grenoblois ne constitue qu'une partie du pôle mondial de compétitivité qui s'étend à d'autres lieux en Isère. Concernant la partie dédiée aux micro et nanotechnologie, le pôle réunit 28400 emplois dont 3900 chercheurs de la recherche publique (CEA, CNRS, INSERM, ...). Le site délivre chaque année 3300 diplômés de l'enseignement supérieur.

Les micro et nano technologies sont en fait le produit du croisement des technologies clés de la biologie santé, des logiciels, des nano sciences, de l'énergie et des matériaux. Il n'y a donc pas à proprement parler de « spécialisation » avec les risques inhérents. 
Du point de vue de l'effort financier, ce sont 4 milliards d'euros qui ont été investi entre 1992 et 2002 et 3 milliards sont prévus d'ici 2007.

Il y a bien un pari sur cet investissement avec des questions fortes d'une part sur les retombées pour les entreprises qui ne sont pas directement partie prenante de ces orientations technologiques mais qui, pourtant, développent de très bons niveaux de technologie (voir par exemple dans le secteur de la mécanique) et d'autre part sur les «dommages collatéraux » dans l'organisation spatiale, les prix du foncier, les perturbations dans la régulation du marché local du travail, etc.

Notons enfin que la Silicon Valley en Californie emploie environ 250000 personnes ce qui ramène le pôle dit de la «Silicon Valley iséroise » à une proportion de $10 \%$ de son modèle.

Sources : AEPI (Agence d'Étude et de Promotion de l'Isère et revue Petites Affiches Lyonnaises, Hors série 2006.

La notion de pôle de compétitivité fait du territoire le cadre organisationnel d'une meilleure articulation entre industrie et innovation, dans la mesure où le territoire est présenté comme la base d'une coopération entre entreprises, laboratoires, universités (production, $\mathrm{R} \& \mathrm{D}$, transfert et formation). Le pôle est défini alors par quatre caractéristiques principales :

- La stratégie de développement économique du pôle doit l'ancrer sur un tissu économique local dynamique, performant face à la concurrence internationale. La cohérence du pôle et de sa stratégie avec l'ensemble plus vaste que constitue le plan de développement économique du territoire, constitue un facteur essentiel.

- Le pôle doit représenter une visibilité internationale suffisante, sur les plans industriels et/ou technologiques. Les projets présentés doivent pouvoir se placer, à terme, dans les premiers rangs mondiaux de leur activité.

- Le partenariat et le mode de gouvernance mis en œuvre sont importants. La qualité et l'efficacité des partenariats de R\&D établis entre les acteurs (industriels, chercheurs, formateurs) sont des critères majeurs de labellisation d'un pôle.

- Les projets à labelliser doivent être créateurs de synergies en matière de recherche et développement, et apporter ainsi des richesses nouvelles à forte valeur ajoutée. L'objectif final est bien d'améliorer la compétitivité de l'offre française sur les marchés internationaux, et donc aussi l'emploi très qualifié.

Sur les 105 candidatures recueillies, le CIADT de juillet 2005 a labellisé 67 pôles et a distingué parmi ceux-ci 6 projets mondiaux et 9 projets à vocation mondiale (16 concernent Rhône-Alpes dont 2 mondiaux). L'enveloppe totale dédiée au financement des pôles prévue est de plus de 1,5 milliard d'euros.

Les pôles ainsi définis s'inscrivent dans une dimension essentiellement urbaine et représentent donc, métaphoriquement, les fonctions urbaines contemporaines dans une phase de réorganisation de la «nouvelle » division internationale du travail (NNDIT). La fonction urbaine a, dans un tel contexte, évolué et rend plausible de tester l'hypothèse d'une « ville qui produit». 


\subsection{L'hypothèse de la ville qui produit}

De multiples signes tendent à montrer la nécessité de revisiter les fonctions économiques de la ville. Au premier rang, on observe que la littérature sur le développement urbain met en relation la globalisation des échanges et les processus de métropolisation autour d'un concept développé par Sassen (1991) : « les villes globales ». Dans un essai récent, Burgel (2006) parle de la revanche des villes comme si ces dernières avaient évolué jusqu'alors vers des formes d'organisation obsolètes et étaient victimes du postmodernisme (ou du moins du post fordisme). Le terme de revanche est sans doute un peu fort dans la mesure où la variété des formes de villes interdit de les enfermer dans une catégorie unique d'où elles seraient issues. Mais il est certain qu'aujourd'hui les villes sont un acteur majeur de la mondialisation et qu'il convient de faire l'analyse de leur place dans les processus de production économique.

Ainsi, dans le processus de globalisation en cours, les rapports production/territoire sont au fondement de l'émergence d'un nouveau visage du capitalisme. Ces rapports se sont affirmés au sortir de la crise du fordisme. Leurs formes évoluent, se diversifient et semblent se déplacer vers le milieu métropolitain. L'économie relationnelle et située va probablement jouer un rôle croissant mais selon des configurations en devenir. La dynamique de développement local spécifique est une forme de développement endogène de valorisation des ressources propres au territoire qui a d'abord été développée en milieu rural lors des fortes poussées de l'exode rural et de la déprise agricole depuis notamment la fin des années soixante dix (du moins dans le cas français) pour gagner maintenant le milieu urbain. La ville produit des ressources spécifiques, une abondante littérature s'est accordée sur ce point.

En effet, ce sont les fondements de la production de richesses qui mutent profondément. La mondialisation crée de l'interdépendance et rend à la fois plus complexe et plus fragile les systèmes techniques. La nécessité de flexibilité rend plus cruciaux les besoins de coopération : «la nouvelle production est relationnelle, elle dépend avant tout de la qualité de coopérations tissées autour des opérations et des projets » (Veltz, 1996). Les territoires comme coalitions d'acteurs visant à la solution de problèmes productifs inédits interfèrent donc dans la constitution, de cette économie relationnelle.

Ainsi, sous l'effet conjugué de l'épuisement du fordisme qui se décentre vers les périphéries et de l'émergence de l'économie de la connaissance (Walliser, 2000)' la territorialisation de la production apparait comme le corollaire paradoxal de la mondialisation. Il reste à approfondir l'analyse de son évolution et de son importance future. En effet, si la territorialisation s'avère une variable permanente de la production, cela entraîne en particulier, une révision importante des contenus des politiques économiques publiques.

Dans la littérature, la ville est traditionnellement pensée comme un producteur d'externalités sans que soient vraiment distinguées les différentes sortes d'externalités. Remy (1966) même « la grande ville en tant qu'incubateur des produits nouveaux » en prenant il y a près de quarante ans, l'exemple de l'industrie électronique nord américaine. Pour lui, « la ville est une condition structurelle de base en vue d'assurer une bonne organisation et une croissance du système économique, tant pour accroître ses capacités productives que pour maximiser le bien être du consommateur final ».

La question est précisée dans les travaux du GREMI (Groupement de Recherche Européen sur les Milieux Innovateurs) qui analysent les villes comme vecteurs d'inno- 
vation technologique et en analyse les conditions. C'est notamment le cas des travaux qui concernent les milieux urbains et l'innovation (Crevoisier et Camagni 2000) où la question de la ville comme productrice de biens et services est développée à travers une déclinaison urbaine du principe du milieu innovateur.

Face à ces perspectives nouvelles dans les années soixante-dix et quatre-vingts mais peu renouvelées depuis, notre hypothèse est bien celle de la ville qui produit et le pôle de compétivité sa forme emblématique.

Les villes produisent non seulement de la valeur économique, mais d'une part la production des villes est plus que la somme des productions de toutes les entreprises qui y sont implantées et, d'autre part, l'intérêt stratégique des villes n'épouse pas nécessairement celui des entreprises qui y sont implantées.

Ainsi, comme y invite l'analyse récente de Bouba-Olga (2006), il s'agit de décrire les nouvelles géographies du capitalisme dans lesquelles, les fonctions d'innovation et la nouvelle production intègrent fortement la variable territoriale. La ville n'est plus seulement le réceptacle de l'activité économique mais elle est productrice en tant que tel. Le collectif d'acteurs qui construit la ville s'autonomise comme acteur économique.

Dans la littérature portant sur l'analyse du phénomène urbain, de sa genèse puis de ses développements, la place de la fonction économique est relativement modeste. La ville parait davantage le lieu et la forme d'organisation de l'habitat qui contient des fonctions économiques mais ce n'est pas la ville en tant que telle qui est un acteur économique. On le comprend d'autant mieux que la rupture libérale de la fin du XVIII siècle avait comme objectif d'autonomiser l'économique par rapport à la société (notamment le politique contrairement à ce qu'en pensaient les mercantilistes). Le souci des analystes était donc d'éliminer la variable spatiale et l'homo oeconomicus n'est ni urbanus ni ruralis, il est toujours le même quelque soit son milieu d'action. La ville ne peut pas être, dans cette conception, un opérateur économique comme une entreprise ou un consommateur. La ville est cependant un «phénomène économique » comme le montrera justement J. Remy (op. cit.) dans la mesure où elle produit des avantages dont vont profiter les acteurs entreprises et consommateurs /habitants.

\subsection{La ville industrielle, contenant de l'activité économique}

L'avènement de la production industrielle s'est fait avec la lenteur d'un siècle entre la Révolution industrielle anglaise dès 1750 et l'avènement du Second Empire en France. Ce processus de généralisation de la production d'objets standardisés et reproductibles aurait pu être strictement lié aux coûts locaux de production et se répartir spatialement au gré de ces coûts. En réalité, les villes ont été les capteurs de l'industrie. Il y a donc, peut être, une nature intrinsèquement urbaine dans l'objet industriel même si la proto-industrie dans les campagnes a été un phénomène important.

Ainsi, on pourra s'interroger sur les rapports entretenus entre une firme et une ville qui sont le plus souvent des rapports de construction mutuelle. Ainsi dans le cas de FIAT à Turin (Manzagol et Jalabert 1999), il est possible d'observer un modèle (au sens de modelage) de la ville par l'activité automobile. La crise de la firme entraîne la fin du mode d'organisation de la grande firme et de ses sous-traitants qui structurent le marché local du travail selon une forme unique. La localisation initiale est due aux aléas historiques 
de l'implantation de la famille Agnelli. Progressivement, se forme une osmose entre la ville et l'entreprise. On serait donc tenté de dire que Turin produit des automobiles. En réalité, c'est FIAT et les sous-traitants qui produisent un bien industriel de type générique c'est-à-dire soumis à la concurrence avec d'autres sites de par le monde sur un produit qui est comparable en tenant compte des effets de gamme. Ce produit répond à une demande standard sur un marché mondialisé pour lequel, l'origine turinoise importe peu et qui fonctionne de façon tout à fait indépendante des conditions spatiales, historiques et culturelles du milieu turinois. Ainsi, la ville offre un certain nombre d'avantages permettant de stimuler l'industrie. En retour, cette dernière apporte prestige et puissance aux villes bénéficiaires de leur présence (cf. au-delà du cas turinois, on peut considérer les cas de Seattle et Boeing ou Detroit et General Motors, par exemple).

Par contre, l'hypothèse de la ville qui produit coïncide avec le constat d'une rupture du modèle de production globalisé considérant l'espace comme une étendue de répartition de l'activité des firmes qui comparent leurs productivités. Dans ce cas, la ville offre bien sûr des avantages mais elle reste essentiellement un contenant d'activité. Or, aujourd'hui, les écarts de coûts de production sont tellement grands que les comparaisons ne sont plus tenables à moins de bâtir des « lignes Maginot » protectionnistes. Il apparaît alors nécessaire de spécifier sa production en fonction du territoire et des coûts qui y sont pratiqués. Le milieu urbain va alors jouer un rôle crucial dans cette spécification. Il est possible de postuler l'émergence d'un véritable tournant territorial qui participe à l'autonomisation de la fonction productive territoriale par rapport à la production des entreprises.

En d'autres termes, dans le modèle productif globalisé traditionnel, la population cible est le salarié et l'institution opératrice est l'entreprise. Les politiques publiques urbaines visant le développement économique sont exclusivement tournées vers la création d'emploi et ce qui est bon pour l'entreprise l'est forcément pour le territoire. La crise de ce modèle se manifeste par l'apparition d'un autre couple où la population cible est l'habitant et l'institution opératrice, le territoire urbain. Dans ce dernier cas, l'entreprise devient un simple truchement pour satisfaire les habitants mais l'accueil d'emplois a cessé d'être l'unique objectif des actions publiques urbaines. L'économie " présentielle » suggérée par Davezies (2005) constitue un exemple intéressant d'autonomisation des stratégies des habitants dans une perspective territoriale. Dans ce cas la raisonnement est simple : il vaut mieux une ville où la richesse vient se dépenser plutôt qu'une ville où la richesse est effectivement produite mais dépensée ailleurs.

\section{Caractéristiques du pôle de compétitivité dans la ville post fordiste}

Le pôle constitue le moyen par lequel se métamorphose la ressource spécifique. On rappelle dans le paragraphe suivant, la notion de spécificité et de métamorphose (Colletis et Pecqueur 2006)

\subsection{Spécificité et gouvernance territoriale}

Les actifs spécifiques ont un coût d'irréversibilité que l'on pourrait appeler aussi un "coût de réaffectation". Cela signifie que l'actif perd une partie de sa valeur productive dans le cas où il est redéployé vers un usage alternatif. Sa formation à une technique 
constitue un coût pris en charge partiellement hors de l'entreprise. Une délocalisation aurait, en partie, un coût de réaffectation pour l'entreprise utilisatrice de cette main-d'œuvre. De même, le capital devient actif spécifique lorsqu'il passe d'une forme d'épargne liquide à une forme de capital investi dans des équipements. Enfin, l'information devient, elle aussi, un actif spécifique lorsqu'elle est élaborée et organisée en vue d'un usage particulier. C'est notamment le cas de l'élaboration de logiciels très techniques concentrant l'information pour servir une production très pointue liée à des usages particuliers.

Les ressources spécifiques ont une nature particulière qui peut être ramenée à quatre caractéristiques.

En premier lieu, ces ressources n'apparaissent qu'au moment des combinaisons des stratégies d'acteurs pour résoudre un problème inédit. Plus précisément, la formulation même du problème en même temps que sa solution fait l'objet d'un processus heuristique marqué par des tâtonnements et des itérations successives.

En deuxième lieu, ces ressources ne sont pas commensurables, ce qui signifie qu'on ne peut les exprimer en prix et qu'elles sont donc absolument non transférables.

En troisième lieu, la nature partiellement hors marché de ces ressources n'est pas incompatible mais complémentaire au marché. Ces ressources résultent d'une histoire longue, d'une accumulation de mémoire, d'un apprentissage collectif cognitif.

En dernier lieu, ces ressources sont, dans certains cas, produites dans un territoire lequel est alors « révélé ». La production de telles ressources résulte en effet de règles, de coutumes, d'une culture élaborés dans un espace de proximité géographique et institutionnelle à partir d'un forme d'échanges distincte de l'échange marchand : la réciprocité. Cette dernière se caractérise par le fait que si l'échange reste "contraint» (obligation sociale), il peut être différé dans le temps (le délai du retour attendu peut être très variable), de plus ce retour n'est pas nécessairement sinon jamais, monétaire. Il peut être confiance, considération, reconnaissance ou savoir. La réciprocité comme terreau des ressources spécifiques est ainsi, le plus souvent, à la base des relations informelles qui composent une « atmosphère industrielle » au sens où l'entendait $\mathrm{A}$. Marshall décrivant certaines concentrations des petites unités industrielles dans l'Angleterre ou l'Allemagne du début du XIX ${ }^{\mathrm{e}}$ siècle. Plus systématiquement, le « sentiment d'appartenance », à un lieu ou à un métier, la « culture d'entreprise » ou encore «l'esprit maison », font partie des ressources « spécifiques », dans un esprit proche de celui de Veblen (1899). En d'autres termes, la rationalité des acteurs loin d'être seulement mécanique, est marquée part les histoires de chaque individu et par les contextes des histoires collectives. La ressource spécifique produite lors d'un processus de construction territoriale, prise globalement, apparaît alors comme le résultat de processus long d'apprentissages collectifs qui aboutissent à l'établissement de règles, le plus fréquemment tacites. De telles règles ont la fonction de trier, de sélectionner et de hiérarchiser l'information, mais aussi d'orienter ou guider les comportements offrant, ainsi à chaque acteur du territoire un espace d'intelligibilité et d'action.

\subsubsection{L'activation et la spécification: un processus de métamorphose}

Le passage de la ressource à un actif spécifique correspond à une stratégie de développement qui peut se décomposer en deux temps : le passage de la ressource à l'actif (génériques), et de l'actif générique à l'actif spécifique. Ces passages doivent être analysés comme des processus particuliers qui font muter en profondeur la nature même des 
objets concernés. C'est dans ce sens que l'on parle de métamorphose comme un changement structurel avec une irréversibilité incomplète selon laquelle on ne peut pas toujours revenir de l'actif vers la ressource et du spécifique au générique en retrouvant l'état initial exact (François, Hirczak et Senil, 2006).

La notion de ressource a été précisée par Hirshman (1986) Selon lui, « il importe moins, pour promouvoir le développement économique, de trouver des combinaisons optimales de ressources et de facteurs de production donnés que de faire apparaître et de mobiliser à son service des ressources et des capacités cachées, éparpillées ou mal utilisées ». En ce sens la ressource se distingue clairement du facteur de production. Cette dernière notion, basique en économie standard pour désigner les ressources qui entrent comme input dans le processus de production, est en effet très restrictive et limitée au cas de l'actif générique dans notre formulation.

Deux cas «d'activation » peuvent être envisagés : celui d'une ressource (générique) potentielle et préexistante, celui d'une ressource (spécifique) virtuelle. Dans le premier cas, le marché est le lieu ou le moyen par lequel se produit l'activation de la ressource. Dans le second cas, la ressource virtuelle peut être activée à la suite d'un processus particulier d'engagement. Ainsi, pour Lévy et Lussault (2003): " une réalité issue du monde physique ou biologique ne peut être ressource que s'il existe un processus de production identifié dans lequel il peut être inséré et qui, par définition, provient de la société (...) Les ressources sont donc toujours inventées (souligné par nous), parfois bien après avoir été découverte comme le pétrole en tant que source d'énergie ou la haute montagne comme gisement touristique ».

Kébir (2004) définit en des termes proches la notion de ressource comme un «méta système mettant en relation un objet (savoir faire, matière première, artefact, etc.) et un système de production produisant un bien ou un service ». Il s'agit d'un « méta système » dans la mesure où il résulte d'une combinaison de deux systèmes préalablement existant : celui de l'objet et celui du système de production ou plutôt, de règles qui le réalise. L'objet/ressource qui va servir de support à la production d'un actif ne peut être réduit à sa dimension d'input mais relève d'un système qui lui est propre : "avant de fournir une planche, un arbre est un arbre. Ceci même lorsqu'il est planté à cette fin » (Kébir,op.cit.). La même perspective peut également être retenue s'agissant des ressources immatérielles que constituent les paysages, les traditions patrimoniales, les savoir faire, voire le capital social. Ainsi l'objet va subir sa métamorphose à travers le système de règles qui le transforme en ressource activée. Le système de production (de règles, selon nous) est le lieu de l'identification (souligné par l'auteur) et de la mise en œuvre des ressources. (...) Elles y sont actualisées, transformées et utilisées (Kébir op. cit.). En d'autres termes, la ressource en devenant actif est transformée par le système de production (pour nous, de règles) et change de nature et au stade de la virtualité, tout peut potentiellement faire ressource mais tout ne peut devenir mécaniquement un actif puisque les conditions de la métamorphose ne sont pas dans l'objet/ressource mais dans les stratégies d'acteurs au sein du processus de production.

Ainsi, les ressources se déploient et se transforment, mais aussi régressent ou redeviennent un potentiel, selon qu'elles évoluent en actifs ou, d'actifs, reviennent au statut de ressources. Cependant le processus de métamorphose qui fait passer la ressource au statut d'actif n'a pas le même sens selon qu'il s'agit de ressources génériques ou spécifiques. 
Dans le cas de la ressource générique, reproductible en tout lieu, ce qui peut faire régresser l'actif de nouveau vers la ressource est uniquement fonction des coûts. On vient de fermer le dernier puits de charbon en Lorraine mais on n'a pas pour autant ôté le charbon. Celui-ci retourne au statut de ressource dans l'état où il était avant l'ouverture de la mine et, dans le cas d'une hypothétique réduction des coûts d'exploitation, cette ressource pourrait redevenir un actif (exploité). On voit bien comment le pôle constitue un opérateur de cette métamorphose qui puise fortement dans l'ancrage territorial. En ce sens, le pôle ne peut être défini par une recherche de productivité mais plutôt de compétitivité par spécification, ce qui est tout à fait différent et nouveau.

\subsubsection{Productivité des pôles, la mal nommée}

A cet égard, la vision des pôles par la DIACT est caractéristique d'une vision qui reste sur un objectif de productivité avec un apport exogène des fonctions urbaines. Il s'agit de partir du phénomène de transformation des productions dans ce que l'on appelle communément l' " économie cognitive » parce qu'elle incorpore une forte quantité de connaissances issue prioritairement de la ville à travers ses institutions productrices de savoir (universités, recherche publique et privée, start up, ...). Pour Jacquet et Darmon (2005) « les économies industrialisées sont engagées dans une nouvelle ère : celle de l'économie de la connaissance, où l'augmentation de la productivité demeure le principal vecteur de croissance (souligné l'auteur), (...) l'avènement de l'économie de la connaissance provient du basculement des modes de production d'un système « tayloriste » (qui consiste à produire en grandes séries pour des marchés restreints, c'est-à-dire le plus souvent nationaux et protégés) à un système nouveau de différenciation et d'individualisation à l'extrême des produits, dans le but de mieux répondre aux attentes hétérogènes des consommateurs (ce qui revient à produire en petites séries pour des marchés devenus mondiaux et concurrentiels) ».

Si nous souscrivons volontiers à l'idée de changement et à la philosophie des pôles de compétitivité, il semble par contre que ce n'est précisément pas sur la productivité que s'adosse la production urbaine (même si celle-là demeure largement une réalité) mais sur la spécificité.

Ces formes, recensées précédemment, montrent que l'on passe d'une offre d'entreprises dans un contexte d'externalités urbaines à une offre de site où l'acteur producteur ne se réduit pas à la somme des entreprises présentes sur le site mais s'étend à tous les acteurs qui, en mettant en mouvement une forme de gouvernance, fabrique le site urbain comme producteur collectif à multiples contributions (y compris générationnelles puisque l'histoire longue compte).

\subsection{Une gouvernance territoriale spécifique pour Grenoble}

Les enjeux que soulèvent les pôles de compétitivité n'ont été abordés que sous l'angle théorique et non dans leur application empirique. Le paragraphe suivant vise à confronter les aspects théoriques au cas concret de Grenoble.

L'agglomération grenobloise a fait l'objet de nombreuses analyses dans la mesure où elle présente une belle réussite dans le domaine des hautes technologies avec des combinaisons originales de stratégies d'acteurs différents (établissements de grandes entreprises, start-up, université, organismes de recherche,...). Cette réussite ne concerne pas la totalité des acti- 
vités de l'agglomération. En effet, d'autres industries plus traditionnelles mais participant de la culture historique de la ville, comme l'agro-alimentaire par exemple, ont pratiquement totalement disparues. Les territoires construits ne concernent pas nécessairement la totalité des populations présentes sur le socle physique du territoire. Il faut raisonner en termes de groupes d'acteurs réunis autour d'une culture partagée représentée à Grenoble par les savoir faire liés aux techniques des semi-conducteurs et des nanotechnologies résultat d'une histoire singulière et longue et de problèmes communs à résoudre.

Robertis (2003) a publié, à la suite d'une longue enquête, une analyse comparative des systèmes locaux de Tricase et Prato en Italie et de Grenoble. Il dresse un panorama des évolutions structurales des villes depuis les années cinquante. Nous reprenons ses résultats concernant l'agglomération grenobloise.

Tableau $\mathrm{N}^{\circ} 1$ : Systèmes locaux de Tricase et Prato en Italie et de Grenoble

\begin{tabular}{|c|c|c|c|}
\hline & marché & organisation & stratégie \\
\hline $50-60$ ’s & $\begin{array}{l}\text { Développement de l'énergie } \\
\text { hydroélectrique, } \\
\text { de l'électrochimie } \\
\text { et de l'électrométallurgie }\end{array}$ & $\begin{array}{l}\text { Grandes entreprises } \\
\text { de type fordiste }\end{array}$ & $\begin{array}{l}\text { Adaptation de l'industrie } \\
\text { traditionnelle aux } \\
\text { nouvelles technologies } \\
\text { disponibles }\end{array}$ \\
\hline 70’s & $\begin{array}{l}\text { Les investissements dans } \\
\text { la recherche et les } \\
\text { innovations } \\
\text { transforment graduellement } \\
\text { les productions. } \\
\text { Les produits traditionnels } \\
\text { pèsent moins }\end{array}$ & $\begin{array}{l}\text { Arrivée des } \\
\text { firmes multinationales et } \\
\text { investissements publics } \\
\text { dans la recherche. } \\
\text { Emergence de } \\
\text { PME spécialisées }\end{array}$ & $\begin{array}{l}\text { Approfondissement du } \\
\text { processus d'adaptation } \\
\text { des structures }\end{array}$ \\
\hline 80 's & $\begin{array}{l}\text { Les produits traditionnels } \\
\text { déclinent. } \\
\text { Une bifurcation s'effectue } \\
\text { sur des produits émergents : } \\
\text { informatique et électronique }\end{array}$ & $\begin{array}{l}\text { Externalisation et } \\
\text { spécialisation approfondie } \\
\text { des PME. } \\
\text { Main d'œuvre plus qualifiée } \\
\text { Salaires moyens à la hausse }\end{array}$ & $\begin{array}{l}\text { Les investissements } \\
\text { dans La recherche } \\
\text { et les innovations } \\
\text { Transforment graduellement } \\
\text { Les productions } \\
\text { Les produits traditionnels } \\
\text { Pèsent moins }\end{array}$ \\
\hline 90’s & $\begin{array}{l}\text { Réduction de la production } \\
\text { de biens matériels. } \\
\text { Grenoble reconnue comme } \\
\text { pôle technologique. } \\
\text { Les grandes entreprises } \\
\text { et les réseaux de PME } \\
\text { se tournent vers l'exportation }\end{array}$ & $\begin{array}{l}\text { Installation d'un système } \\
\text { spatial du type « core and } \\
\text { ring » : autour } \\
\text { des multinationales gravitent } \\
\text { des PME performantes et } \\
\text { ancrées dans la recherche }\end{array}$ & $\begin{array}{l}\text { La baisse des investissements } \\
\text { publics (politique nationale) } \\
\text { impose de chercher de } \\
\text { nouvelles solutions pour } \\
\text { maintenir le leadership du } \\
\text { site (MINATEC) }\end{array}$ \\
\hline
\end{tabular}

D'après Robertis (2003)

Ce panorama appelle quelques commentaires relatifs aux processus de gouvernance : L'activité décrite a une unité dans la mesure où il s'agit de l'industrie issue de l'hydroélectricité mais elle n'est que partiellement représentative de l'agglomération grenobloise puisqu'elle ne recouvre qu'une partie de l'industrie elle-même (au-delà de l'agro-alimentaire évoqué plus haut, d'autres activités sont importantes dans la ville : mécanique, matériaux, éléments pour l'aéronautique, etc.). 
Le territoire des nouvelles technologies inscrit son évolution dans le temps et l'on a affaire à une trajectoire de bifurcation qui infléchit les productions, les manières de produire et les stratégies collectives dans le sens d'une adaptation du territoire à la globalisation.

La constitution d'un « dedans » (la communauté scientifique, industrielle et universitaire grenobloise) et d'un « dehors » (le marché international des semi-conducteurs et des nanotechnologie) est bien, dans ce cas grenoblois, l'expression d'un processus de spécification des actifs qui discrimine fortement cette communauté par rapport au reste de la population et par exemple vis-à-vis des branches traditionnelles évoquées plus haut.

Il est possible d'évoquer, pour Grenoble, un modèle mixte de gouvernance tourné vers la maîtrise des hautes technologies. Il bénéficie d'une combinaison de financements publics et privés, de recherche publique, de grandes entreprises privées et de petites entreprises issues du territoire. Les conditions d'équilibre entre le public et le privé se modifient dans le temps comme l'a montré le tableau ci-dessus mais s'appuie sur l'élaboration de réseaux cognitifs locaux élaborés dans le temps long (Bernardy de Sigoyer et Boisgontier, 1988). Le professeur L. Néel (Prix Nobel de Physique et un des hommes clés de la construction institutionnelle) dans la préface de l'ouvrage de Bernardy de Sigoyer et Boisgontier rappelle le caractère endogène du développement grenoblois : "l'initiative locale a été la base de beaucoup de créations, que ce soit à l'Université avec des centres de recherche, ou dans l'industrie (...) La région parisienne n'y a jamais parachuté d'industries importantes ni créé dans un désert des succursales de ses laboratoires de recherche ». En matière de combinaison et de mixité, une des grandes caractéristiques du pôle grenoblois est sa non spécialisation, mais le croisement de technologiques produisant des « métatechnologies » qui fournissent la matière de la spécificité du lieu.

En définitive on peut sans doute parler pour Grenoble de District Technologique à l'instar des districts industriels marshalliens tels que les ont redéfinis les économistes italiens (G. Becattini 1979) ${ }^{1}$. L'attribution du label de pôle mondial de compétitivité comme défini plus haut correspond bien aux caractéristiques grenobloises et démontre que le processus de spécification constitue un moyen d'observation pertinent pour ordonner une analyse des gouvernances territoriales.

\subsection{Linéaments du modèle urbain des pôles de compétitivité}

Quatre éléments paraissent constituer les base caractéristiques du modèle : l'avantage différenciatif, la ressource d'amont, l'ancrage territorial et le rôle nouveau de la demande.

\subsubsection{L'avantage différenciatif}

La délocalisation individuelle comme tendance illustrative de la globalisation tend à jouer sur un mécanisme préfordiste de recherche de coûts de production faibles en séparant à nouveau les populations productrices des populations consommatrices. Cette projection dans un monde où les cadres de l'échange sont de moins en moins les Etats-Nations mais

${ }^{1}$ G. Becattini (1979) : Dal settore industriale al distretto industriale, Rivista di Economia e Politica Industriale, vol. 2, pp. 7-21. On notera également la tentative intéressante de mettre en évidence des systèmes productifs urbains qui croisent les qualificatifs des cités et des clusters, donnant ainsi le mot « clusties »: F. Gaschet et C. Lacour : Les systèmes productifs urbains : des clusters aux « clusties », multigraphié IERSOGRES-IFREDE, 2006, Bordeaux, 25 pages. 
plutôt les « régions », c'est à dire des configurations agglomérées de grande taille mais construites et non données, remet en cause le modèle type ricardien de l'échange international à base d' »avantages comparatifs ». Lorsque les producteurs de chaussures du choletais nous disaient (Courlet et Pecqueur, 1992) que leurs concurrents d'Asie du Sud-est mettaient leurs chaussures sur le marché français au prix où, eux les choletais, achetaient le cuir (!), on atteint là les limites de la comparabilité. Dans ce cas comme dans beaucoup d'autres dans l'économie contemporaine, la solution au problème consiste à différencier le produit pour qu'il devienne « spécifique » et donc échappe à la concurrence. Les fabricants du choletais ont donc cherché tout d'abord à monter en gamme pour permettre une augmentation des prix, puis dans un second temps à maîtriser la mode en amont du produit afin de faire ainsi un produit complètement différent de la chaussure standard importée. Enfin, les réseaux d'entrepreneurs localisés ont abandonné le lien d'un produit commun pour aborder ensemble le marché des jeunes de 10 à 25 ans (chaussure, prêt-à-porter, brioches et bonbons, services, etc.). Cette différenciation a été obtenue par l'ensemble des acteurs dans la mesure où l'acteur public y participe. Les acteurs mobilisent les savoir faire accumulés et s'éloignent de la conception des districts organisés autour d'une spécialisation industrielle. Le principe de l'avantage différenciatif qui se distingue de la seule spécialisation apparaît donc comme la première caractéristique du modèle.

\subsubsection{La ressource comme un amont de l'input}

L'analyse économique courante s'en tient le plus souvent, aux inputs directement présents et disponibles pour être combinés dans une opération productive. Une caractéristique majeure du modèle de la ville qui produit, est précisément de remonter à l'amont de l'actif pour considérer toutes les ressources comprenant notamment celles qui ne sont que virtuelles n'ayant pas d'existence préalable à l'action de les faire naître.

Si l'existence du territoire suppose une construction historique longue de la part des acteurs cela signifie que les externalités territoriales créées par les acteurs résultent d'un processus amont dans un monde non marchand. C'est le sens d'une distinction cruciale entre ressource et actif. L'actif est un facteur « en activité » c'est à dire valorisé sur le marché. La ressource, à la différence de l'actif, constitue une réserve, un potentiel latent voire virtuel qui peut se transformer en actif si les conditions de production ou de création de technologie le permettent. Ainsi, l'expression de la variable territoriale est elle à chercher dans les conditions d'élaboration de ressources dans cet amont qui est l'expression du travail d'une société et plus précisément du territoire sur lui-même et sur la manifestation de son identité. C'est ainsi que les villes mobilisent pour leur production spécifique, des ressources cognitives et/ou culturelles qui seront le fondement (souvent aléatoire) de leur développement futur.

\subsubsection{L'ancrage territorial ou de l'importance de la proximité géographique}

L'établissement d'une firme peut être considéré comme l'unité productive de base d'une économie. Il est « au carrefour d'une triple insertion : dans son groupe industriel, dans sa branche et dans son territoire. Les stratégies des firmes les amènent à qualifier respectivement chacun des trois modes d'insertion » (Zimmermann, 1998) Face à ces choix, la firme privilégiera une insertion plutôt qu'une autre, en fonction de la nature des ressources qu' elle cherche à utiliser, on trouve les territoires d'implantation. Ceux-ci ne 
sont plus des espaces passifs d'accueil anonyme d'une activité productive. Le territoire est, en effet le résultat d'une création par les acteurs. Dans ce cas, une première logique peut apparaître, celle des offres concurrentielles d'avantages génériques. On est alors confronté à une concurrence classique (mais aussi féroce) de territoires sur un "marché des sites ». Les territoires visent alors à valoriser des avantages triviaux (gratuité du foncier, niveau général des salaires bas, exemptions de taxes, etc.). cependant, l'exacerbation de ce type de concurrence ajouté aux phénomènes d'innovation territorialisée par différents types de clusters ou par les villes (voir notre première partie), implique l'élaboration d'une offre spécifique de site beaucoup plus spécifique et visant à échapper aux effets pervers de la concurrence des sites.

Ce sont, donc bien deux modèles productifs encastrés qui apparaissent et cohabitent en fonction du type de combinaison noué entre la proximité organisationnelle et la proximité géographique montrant ainsi l'irruption claire de la variable territoriale dans l'organisation du capitalisme contemporain.

\subsubsection{Le rôle nouveau de la demande}

Le phénomène de différenciation des produits se fait par la qualité et apparaît donc comme très cohérent avec le contexte post-fordiste. Il a pour objectif de donner les moyens aux producteurs et aux transformateurs de mettre sur le marché des produits dont la qualité et la spécificité sont identifiables et reconnus et de disposer ainsi d'un outil nouveau de compétitivité en jouant non plus sur les seuls coûts de production mais sur la qualité. A cette qualité demandée par les consommateurs s'associent l'idée d'une mise en relation de divers biens à travers leurs caractéristiques (Lancaster -1991) et donc la question de la combinaison possible de ces biens. Des biens différents peuvent donc être reliés par une ou plusieurs caractéristiques communes ce qui leur confère une «identité partagée » et donc une complémentarité que l'on retrouve, par exemple, dans des produits de même origine ou les services touristiques liés à un même lieu. On peut donc voir se développer une offre de site urbain largement définie par le mouvement de la demande qui façonne l'offre spécifique.

\section{Conclusion}

Depuis l'archipel de Veltz et les régions de Scott, on a avancé dans l'hypothèse plus radicale de la «clusterisation » de la nouvelle division internationale du travail. BoubaOlga (2006) propose de décliner l'idée de « petits mondes » développée notamment par J.B.Zimmermann (2002). « Cette économie de petits mondes produit une géographie particulière des activités, où se combinent un réseau dense de relations locales (techniques, économiques, sociales, institutionnelles et financières) au sein, pour l'essentiel, des agglomérations, et un réseau - sans doute moins dense mais très structurant- de relations (techniques, économiques, financières,...) globales. Cette économie se traduit par un creusement des inégalités, au profit des détenteurs de capital financier et de capital (humain ou physique) spécifique et au détriment évident des acteurs qui ne disposent pas de capital, ou seulement d'un capital générique ». On a là une belle métaphore des clusters présentée comme des noyaux isolés et pour lesquels nous pensons à des effets restructurants et régulateurs beaucoup plus forts, mais là est le débat. 
On ressent donc le besoin d'élargir les premières intuitions sur les SPL (eux mêmes étant une tentative de généralisation des Districts Industriels) pour produire des typologies (Grossetti, 2003) adaptées aux réalités de la globalisation. Mais au delà d'une analyse de formes nouvelles, l'ambition esquissée ici, va donc jusqu'à l'élaboration d'un modèle de conduite de la globalisation par les dynamiques territoriales.

\section{Références}

Becattini G. ,1979, : Dal settore industriale al distretto industriale, Rivista di Economia e Politica Industriale, vol. 2, pp. 7-21.

Bernardy de Sigoyer M. et Boisgontier P., 1988, Grains de Technopole : micro-entreprises grenobloises et nouveaux espaces productifs, Presses Universitaires de Grenoble.

Blanc C., 2004, Pour un écosystème de la croissance, rapport au Premier Ministre, Assemblée Nationale, 78 pages.

Bouba-Olga O., 2006, Les nouvelles géographies du capitalisme, collection Economie Humaine, Le Seuil.

Bouba-Olga O., 2006, Les nouvelles géographies du capitalisme, Le Seuil.

Burgel G., 2006, La revanche des villes, Paris, Hachette.

Courlet C. et Pecqueur B., 1992, Les systèmes industriels localisés en France : un nouveau modèle développement, in Benko G. et Lipietz A. (dir.), Les régions qui gagnent, Presses Universitaires de France, pp. 81-102.

Crevoisier O. et Camagni R., 2000, Les milieux urbains : innovation, systèmes de production et ancrage, IREREDES, Neuchâtel.

Davezies L. 2005, Vers une macroéconomie locale. Le développement local entre économie productive et présentielle, DATAR.

Robertis de S., 2003, Spazio produzione regolazione : strategie post( ?)fordiste nei sistemi locale di Tricase, Prato e Grenoble, F. Angeli, Milano, 135 pages

François. H., Hirczak M. et Senil N., 2006, Territoire et patrimoine : la co-construction d'une dynamique et de ses ressources, Revue d'Economie Régionale et Urbaine, n5, pp. 683-700.

Grossetti M., 2003, Concentration d'entreprises et d'innovation ; esquisse d'une typologie des systèmes productifs locaux, Géographie, Espace, Société, volume 6, n², PP. 163-178.

Gumuchian H. et Pecqueur B., 2007, La ressource territoriale (eds), éditions Anthropos, Economica.

Hirshman A.-O., 1986, Vers une économie politique élargie, Editions de Minuit

Jacquet N. et Darmon D., 2005, Les pôles de compétitivité, le modèle français, Paris, La documentation française.

Kebir L., 2004, Ressource et développement : une approche institutionnelle et territoriale, thèse de doctorat en Sciences économiques, réalisée à l'Université de Neuchâtel, Neuchâtel.

Lancaster K., 1991, Modern Consumer Theory, Edward Elgar, New York,.

Lévy J. et Lussault M., 2003, Dictionnaire de la géographie et de l'espace des sociétés --notice « ressource »-, Belin, 1003 pages.

Mac Luhan M., 1970, Guerre et paix dans le village planétaire, Paris, Robert Laffont,

Manzagol C. et Jalabert G., 1999, Technopoles d'Europe et d'Amérique du Nord, in Fontan J.M., Klein J.L. et Tremblay D.G., Entre la métropolisation et le village global, Presses de l’Université du Québec, pp ; 159-174.

Ohmae K., 1995, The End of the Nation-State: The Rise of Regional Economies, New York, The Free Press.

Pecqueur B., 2006, De la ville qui consomme à la ville qui produit. La reterritorialisation des fonctions économiques de l'urbain, Annales de la Recherche Urbaine, $\mathrm{n}^{\circ} 101$, novembre, pp. 7-14

Remy J., 1966, La ville : phénomène économique, Bruxelles, Les Editions Ouvrières.

Sassen S., 1991, Global Cities, Princeton University Press. 
Veblen T, 1899, (1970 pour la trad.fr.), La théorie de la classe de loisir, Gallimard

Veltz P., 1996, Mondialisation, villes et territoires. L'économie d'archipel, Paris, Presses Universitaires de France, collection Economie en Liberté.

Walliser B., 2000, L'économie cognitive, Editions O. Jacob, Paris.

Zimmermann J.B., 2002, «Grappes d'entreprises » et «petits mondes : une affaire de proximité », Revue Economique, vol. 53, n³, mai, pp. .517-524. 